Neurosurg Focus 22 (6):E27, 2007

\title{
Peroneal intraneural ganglia
}

\author{
Part II. Lessons learned and pitfalls to avoid for successful diagnosis and \\ treatment
}

\author{
Robert J. Spinner, M.D., ${ }^{1,2}$ Nicholas M. Desy, B.Sc., ${ }^{1,4}$ Michael G. Rock, M.D., 2 \\ AND Kimberly K. AMrAMi, M.D. ${ }^{3}$
}

\author{
Departments of ${ }^{1}$ Neurologic Surgery, ${ }^{2}$ Orthopedics, and ${ }^{3}$ Radiology, Mayo Clinic, Rochester, \\ Minnesota; and ${ }^{4}$ McGill University School of Medicine, Montreal, Quebec, Canada
}

\begin{abstract}
$\checkmark$ The authors describe common modes of failure in the diagnosis and treatment of patients with peroneal intraneural ganglia. Illustrated examples correlate the modes of failure and the diagnostic or surgical errors. Understanding these pitfalls reinforces the rationale behind current treatment recommendations as outlined in the companion article. Avoiding these pitfalls will ultimately improve outcomes.
\end{abstract}

KEY WORdS - cyst - intraneural ganglion - peroneal nerve - recurrent disease • superior tibiofibular joint

I

N Part I of this two-part review, we discussed our current strategy for diagnosing and treating patients with peroneal intraneural ganglia. ${ }^{11}$ In this paper we will illustrate common modes of failure in patients treated at our institution and elsewhere to demonstrate the ubiquitous nature of the problem. Based on our comprehensive review, we correlate these treatment failures with the principles proposed to improve outcomes. We introduce these concepts based on the most common type of intraneural ganglion that forms within the peroneal nerve originating from the superior tibiofibular joint. ${ }^{8}$ Nevertheless, the same principles can be extrapolated to other nerves arising at other sites.

\section{General Pitfalls}

In our opinion, the majority of diagnostic and treatment errors relate to a lack of knowledge of and inexperience with the underlying pathogenesis of this rare condition on the part of radiologists and surgeons who are unfamiliar with the anatomy and pathological features of this disease. Although diagnostic and imaging pitfalls lead to surgical failures, such failures can still occur despite accurate preoperative diagnoses if interventions do not completely address the underlying pathoanatomy.

\section{Diagnostic Pitfalls}

A common error rests in the failure to diagnose a mass

Abbreviations used in this paper: FSE = fast spin echo; $\mathrm{MR}=$ magnetic resonance. lesion prior to surgery by relying exclusively on physical examination. In our opinion, when patients present with predominantly deep peroneal nerve lesions manifested by a foot drop, a peroneal intraneural ganglion should be suspected. Patients with this type of presentation whose symptoms localize to the deep peroneal nerve should undergo imaging of the region, including the common peroneal nerve and its branches. Although the diagnosis of an intraneural cyst can certainly be established at operation without preoperative imaging, for those less experienced with this entity, appropriate preoperative imaging studies will allow further review and preparation prior to surgery, including identification of the requisite joint connection. If necessary, a referral to a specialist experienced in the treatment of these cysts can be made after a complete clinical and radiological evaluation.

\section{Imaging Pitfalls}

Even if preoperative imaging is performed, the definitive diagnosis of an intraneural cyst is rarely made prospectively. Its full proximal and distal extent may not be revealed with conventional imaging, which may not allow identification of the joint connection or appreciation of the length of the cyst. Furthermore, an intraneural cyst is often misdiagnosed as an extraneural cyst or even as a solid lesion such as a schwannoma.

The largest potential imaging pitfall is the failure to consider that these cysts arise from a joint. Not identifying the joint connection is based on a lack of visualization (not seeing it), and/or a lack of appreciation of the pathoanatomy (not looking for it). The solution is to have knowledge of 


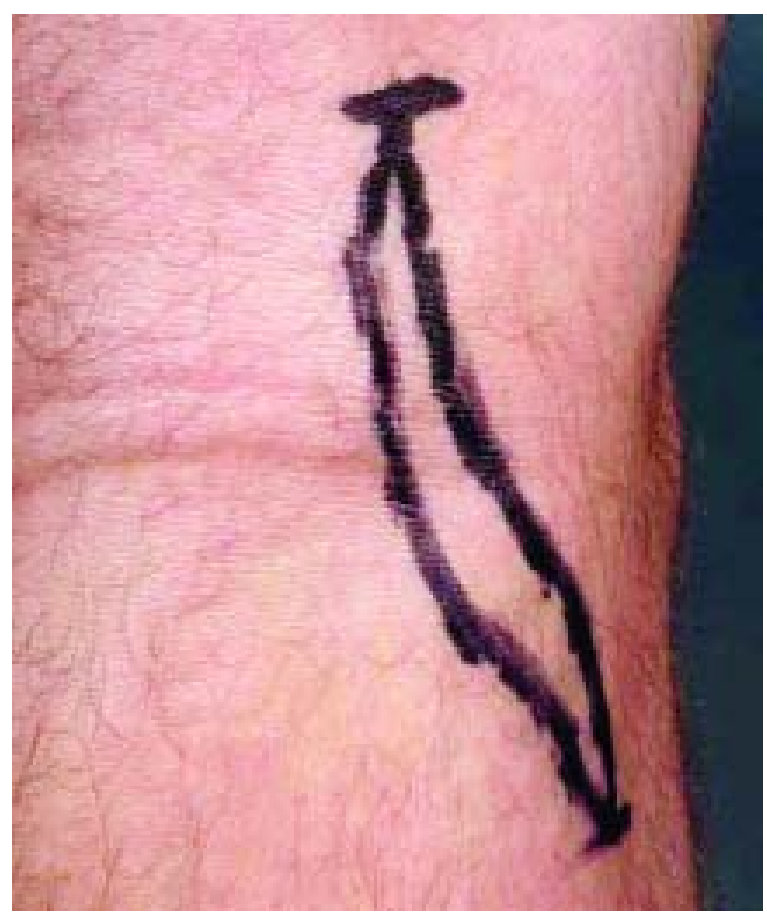

FIG. 1. Preoperative photograph showing an example of failure to visualize the peroneal intraneural cyst's origin from the superior tibiofibular joint on ultrasound. In our opinion, Fig. E-1 in this recent article on the Neurology Web site that was included as representative of the full extent of the cyst (and to help gauge surgical intervention) is misrepresentative. The area of the cyst outlined in magic marker on the proximal leg does not extend anteromedially enough to connect the classic U-shaped (cystic) articular branch (see Spinner, et al., Neurosurgery 59: 157-166, 2006) over the anterior surface of the fibula, and the superior tibiofibular joint. (Reproduced with permission from Visser LH: High-resolution sonography of the common peroneal nerve: detection of intraneural ganglia. Neurology 67:1473-1475, 2006.).

this articular branch connection and to perform imaging studies that will be at a high enough resolution to confirm this by identifying the joint connection definitively., ${ }^{4,9,-12}$ This requires full- or high-field magnets to achieve the necessary signal-to-noise ratio; open or low-field magnets cannot provide similar results without very extended imaging times. Using large fields of view, thick sections, or the wrong coil will all compromise spatial resolution and limit the ability to see what are often very tiny connections. Another common error is failing to use fat suppression on the T2-weighted images or appropriate T2 weighting that will allow the contrast resolution necessary to identify subtle signal changes in the nerves and muscles that are affected. Contrast agents should be used in every case, with the only exception being follow-up examinations obtained at short intervals during which the changes in underlying pathological features are not significant. Despite published reports claiming no postoperative recurrence (see Krishnan and Schackert, Fig. 3), postoperative examinations should follow the same principles; otherwise, recurrent cysts may not be discerned.

The most obvious avoidable error independent of specific imaging parameters is not seeing the full extent of the disease: not imaging the joint of origin itself (Figs. 1-3) or fail-

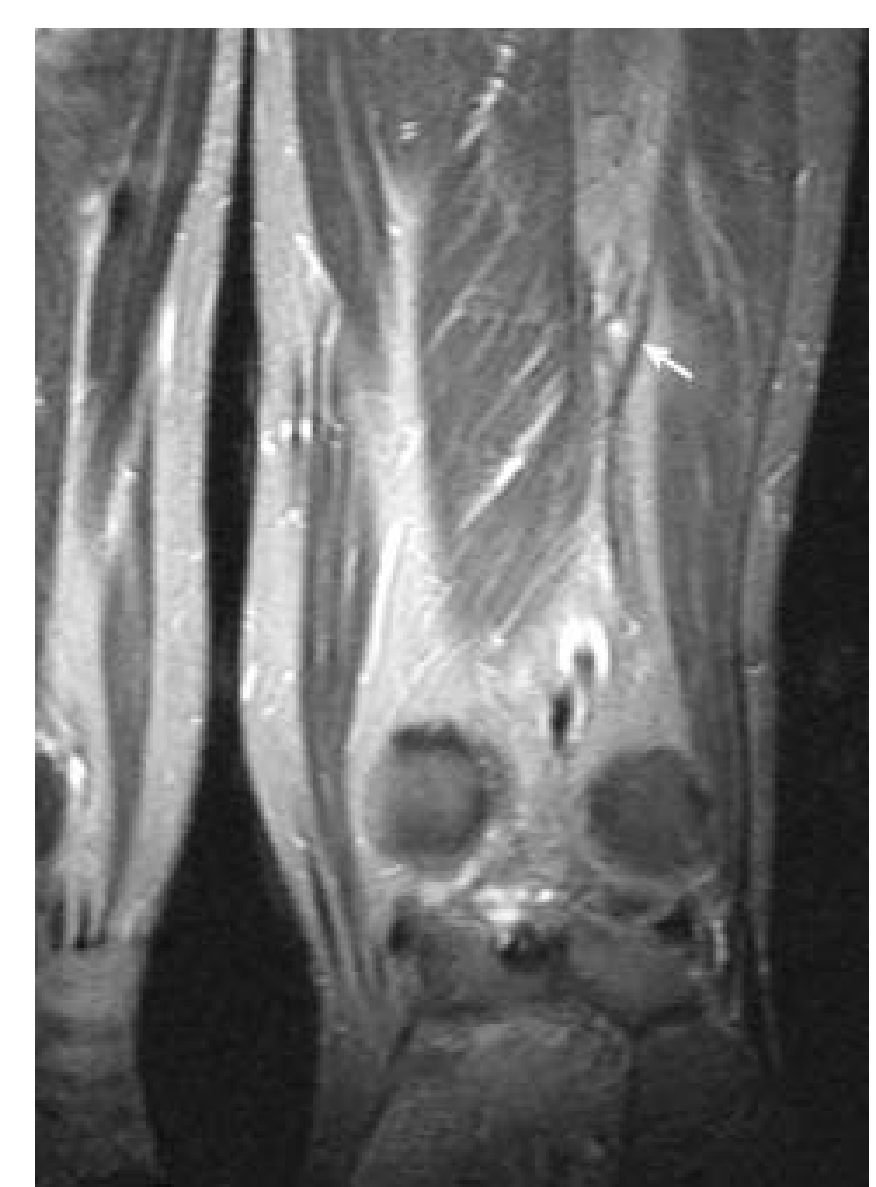

FIG. 2. Example of failure to visualize the tibial intraneural cyst's origin from the superior tibiofibular joint on MR imaging. Krishnan and Schackert ${ }^{1}$ concluded that this tibial intraneural ganglion cyst arose de novo within the sciatic nerve far from a joint. Reinterpretation revealed the true nature of the cyst's derivation from the posterior aspect of the superior tibiofibular joint. The coronal T2-weighted FSE image with fat suppression reveals cystic change within the distal sciatic nerve in the midthigh (arrow). Because of the technical parameters used on this examination, the knee joint and cyst at the superior tibiofibular joint ${ }^{12}$ are suboptimally imaged. The cyst originating from the superior tibiofibular joint could only be visualized with image reconstruction and is not included on this MR image but is reported in another publication (see Spinner et al., Skeletal Radiol 36: 281-292, 2007). ${ }^{12}$

ing to include the entire extent of the lesion (Fig. 4). Crosssectional imaging is usually targeted to a specific clinical problem, and in cases in which the possibility of an intraneural cyst is not considered, the appropriate or complete area may not be imaged. An example of this would be subclinical involvement of the tibial nerve in cases in which a peroneal intraneural cyst arose from the superior tibiofibular joint and ascended to the sciatic nerve, where it crossed over and then descended down the terminal branches. ${ }^{6} \mathrm{Al}-$ though incomplete visualization of an area is common with MR imaging, it is possible with any imaging modality, most notably with ultrasonography, in which the field of view is restricted based on the operator's impression of the extent of the problem (Fig. 1). It is tempting to center the image on the cyst itself, but this often results in exclusion of the lesion's origin and full proximal and distal extent from the 


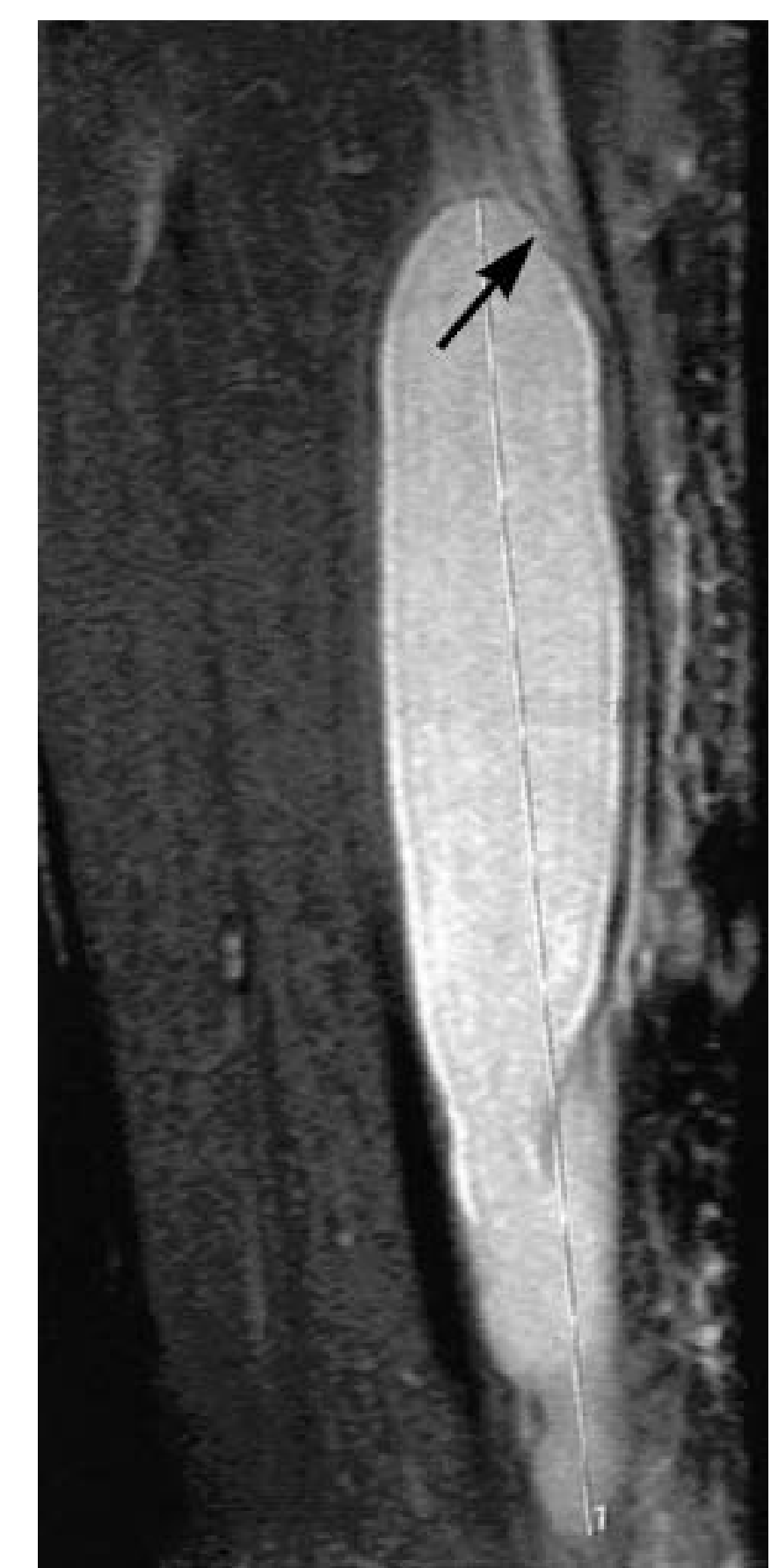

FIG. 3. Example of failure to visualize the tibial intraneural cyst's origin from the ankle region. Previously published sagittal T2weighted MR image of the leg with fat suppression fails to demonstrate the full longitudinal extent of the cystic structure seen, specifically neglecting to include the ankle joint as a possible site of origin for the cyst. The authors have called attention to the displaced vessels (arrow). (Reproduced with kind permission of Springer Science and Business Media; originally Fig. 1 in Adn M, Hamlat A, Morandi X, Guegan Y: Intraneural ganglion cyst of the tibial nerve. Acta Neurochir [Wien] 148:885-889, 2006.) On reinterpretation of this MR image it was suggested that the tibial intraneural ganglion within the tarsal tunnel arose from a joint in the ankle region that was not visualized (see letter to the editor by Spinner and Amrami ${ }^{3}$ in Acta Neurochir [Wien], 2006).

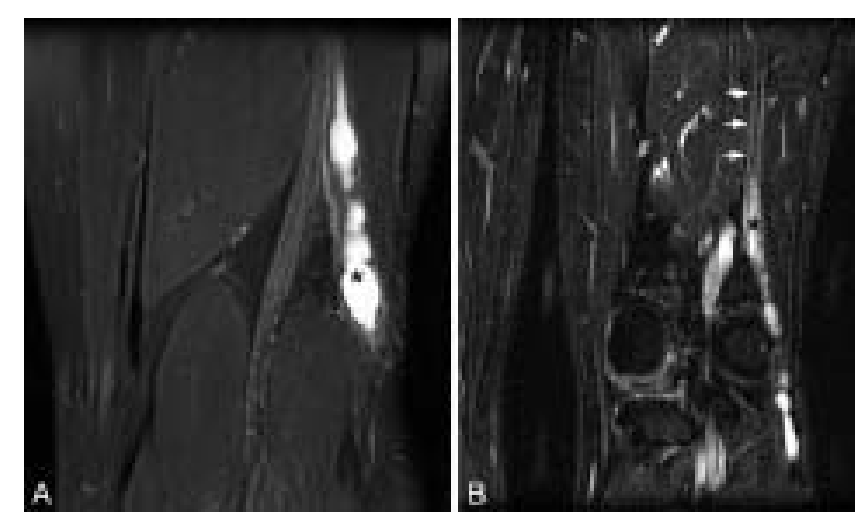

FIG. 4. Failure to see the full extension of the peroneal intraneural cyst into the sciatic nerve on MR images. A: Coronal T2weighted image with fat suppression shows a tubular cystic structure within the peroneal nerve (asterisk) at the lateral knee. On this image neither the proximal nor distal extent of the cyst is displayed. The visualized portion of the cyst begins above the knee with its origin from the superior tibiofibular joint only implied by the course of the cyst along the common peroneal nerve. B: Coronal T2-weighted FSE image with fat suppression (anterior to panel A) demonstrates the primary ascent of the peroneal intraneural cyst (asterisk) within the peroneal division of the sciatic nerve (arrows). The jet streaks (arrows) are above the level visualized in panel A. The full sciatic nerve extension of this cyst was not appreciated prospectively by our group and could only be appreciated in retrospect on this sequence. The superior tibiofibular joint connection not seen in this particular image was demonstrated in Fig. 2 in a previous report. ${ }^{8}$

field of view. Inclusion of the entire knee and superior tibiofibular joint to below the fibular neck is critical because identifying and treating the cyst's origin allows definitive treatment of the lesion, decreasing the possibility of recurrence after surgery. Additionally, cysts may be complex, with adventitial, intramuscular, or even intraosseous extension. Imaging the complete cyst is important to understand the pathoanatomy and to ensure effective therapy.

\section{Surgical Pitfalls}

Errors related to surgery on peroneal intraneural cysts are understandable. The role of the articular branch may not be known, the distal anatomy may be unfamiliar, and the surgery can be technically difficult. Understanding the pathogenesis of these cysts allows a systematic approach to their surgical management. The surgical strategy that we recommend $^{11}$ (that is, dissection of the articular branch to the superior tibiofibular joint, disarticulation of the superior tibiofibular joint, decompression of the cyst, and disconnection of the articular branch) facilitates neural recovery, minimizes intraneural dissection, and in our opinion, decreases risks of recurrence. Failure to perform these steps can lead to predictable adverse consequences (Fig. 5).

Dissection. In our experience, the most common surgical error is the failure to identify the joint connection. Surgeons may not be aware of the diseased articular branch and its role in the pathoanatomy. The more expansile cyst is easi- 


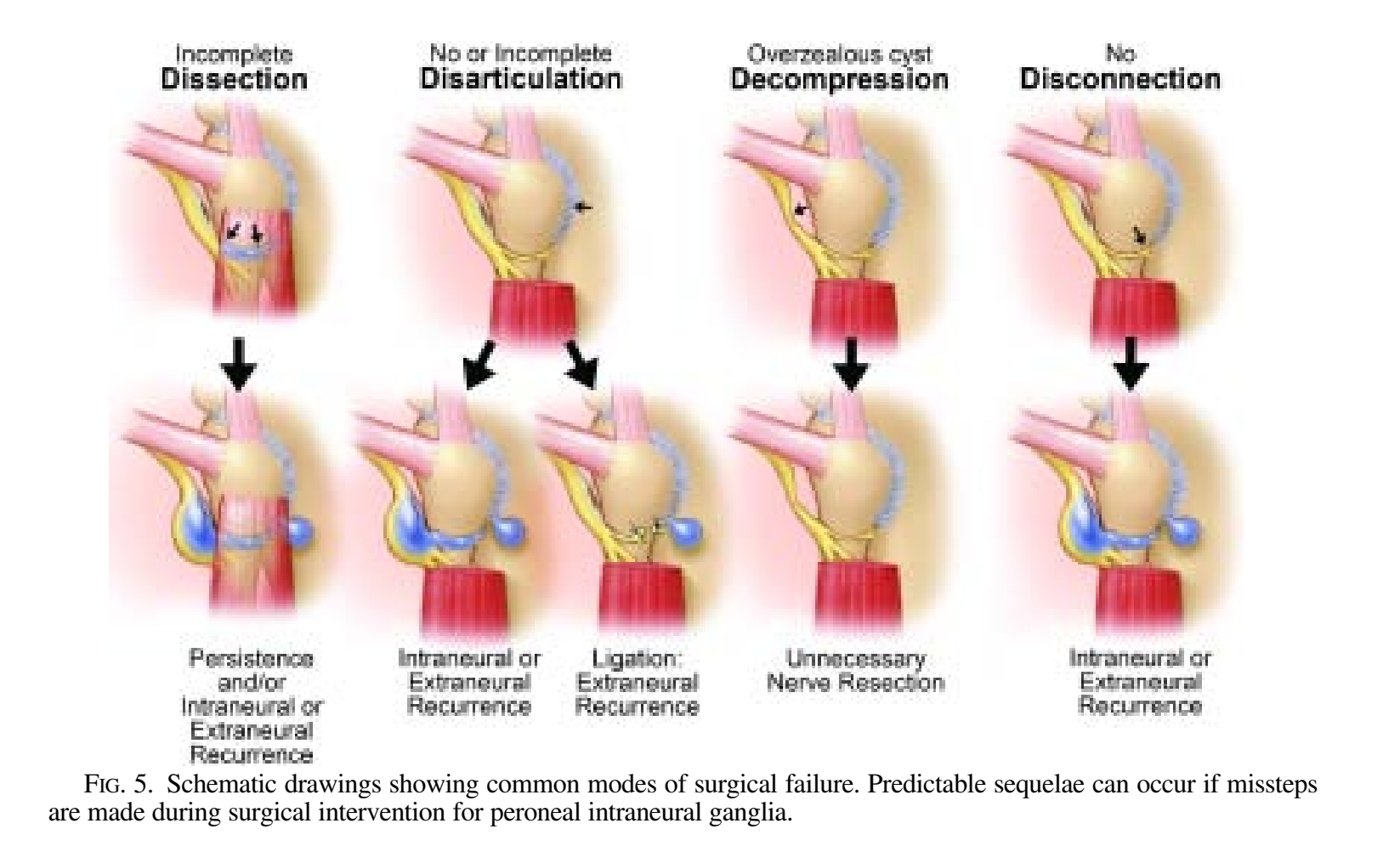

ly seen, and the extension of the lesion along the articular branch may not be sought or may be seemingly occult on visual inspection. The surgical dissection must display the full anatomy and trace the articular branch to the point where it penetrates the superior tibiofibular joint capsule. If the articular branch is not identified and treated, persistence of the joint connection with residual cyst will occur, with the potential for recurrence of the original cyst or even extension beyond its original dimensions. We have demonstrated these phenomena in patients thought to have undergone "gross total resection" and in patients who have made a good but incomplete clinical recovery in the initial postoperative period. ${ }^{5,7}$

Disarticulation. The intrinsic problem relates to a degenerated superior tibiofibular joint and a defect in the joint capsule. If the joint's synovium (which is responsible for the production of joint fluid) is not removed completely, the problem can recur. Curetting the joint or incomplete joint resection may not adequately eliminate the synovium. Depending on the path of least resistance, this can lead to cyst reaccumulation either extraneurally (Fig. 6) or intraneurally. The possibility of intraneural recurrence can be eliminated with disconnection of the articular branch. Because a more global degenerative process exists that may also affect the neighboring knee joint, extraneural cyst may occur at the knee following superior tibiofibular joint resection.

Care must be taken during the exposure of the superior tibiofibular joint to maintain the lateral collateral ligament, lest instability occur. Injury to the anterior tibial vessels can occur with deep joint resection. Although we favor resection of the joint of origin, namely the superior tibiofibular joint in cases of peroneal and tibial intraneural ganglia, we wish to emphasize that such joint resections should not be performed for intraneural cysts occurring at other sites.

Decompression. Decompression by itself is not a defini-

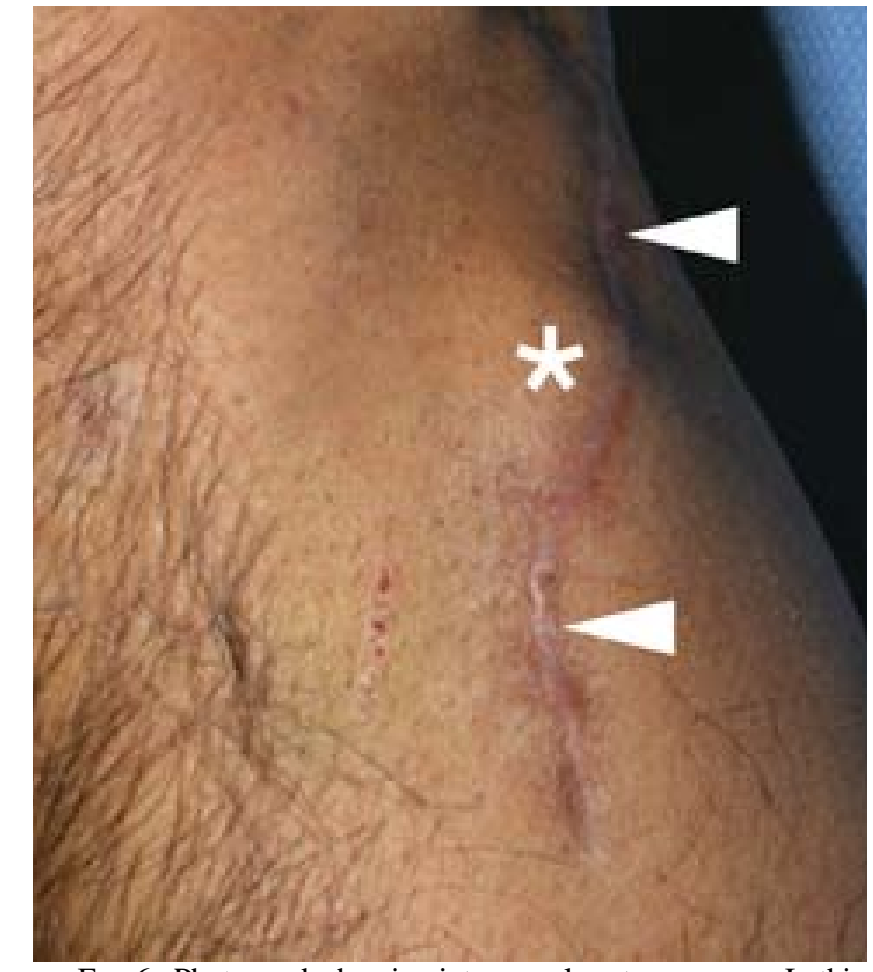

FIG. 6. Photograph showing intraneural cyst recurrence. In this patient, two previous decompressions of his peroneal intraneural ganglion had failed. The visualized soft tissue swelling (asterisk) in the previous surgical wound (arrowheads) corresponds to the recurrent intraneural cyst. Neither preoperative MR imaging nor surgical dissection revealed the superior tibiofibular joint connection. (Reprinted with kind permission of Springer Science and Business Media; originally Fig. 1 in Spinner RJ, Amrami KK, Rock MG: The use of MR arthrography to document an occult joint communication in a recurrent peroneal intraneural ganglion. Skeletal $\mathrm{Ra}$ diol 35:172-179, 2006). 
tive solution to treat this clinical entity when the synovium and joint connection remain intact. This is easily understandable given the joint-related problem. A long experience with percutaneous aspiration of more common (extraneural) cysts and even with intraneural ganglia has demonstrated predictable recurrences with this technique. Furthermore, blind aspiration can result in direct nerve injury and should absolutely be avoided. Similarly, surgical decompression without attention to the articular branch and joint is also doomed to failure. This mode of failure leading to intraneural recurrence has been well described in the literature (Fig. 7), ${ }^{7,8}$ and is illustrated in the case detailed in the companion paper. ${ }^{11}$

Overzealous attempts at resecting the entire cyst and even the cyst wall have been recommended by others. In some cases, the entire peroneal nerve has been resected and nerve grafts used. We believe that our less invasive technique of targeted intervention minimizes the need for intraneural dissection and decreases the risk of iatrogenic neural injury.

Disconnection. If the articular branch is not disconnected from the superior tibiofibular joint, intraneural recurrence is inevitable. To our knowledge, there has never been an intraneural recurrence when the articular branch has been completely disconnected, resected, or ligated (further supporting the articular theory). To avoid further denervation of the tibialis anterior muscle, we preserve the distal branch to this muscle.

A portion of the disconnected articular branch is submitted as a specimen for pathological investigation. An experienced pathologist or neuropathologist can distinguish this type of ganglion cyst from other cystic lesions, such as cystic schwannomas. In addition, he or she can help confirm the diagnosis of an intraneural cyst (when necessary, using a variety of stains and nerve-specific antibodies to help establish the presence of cyst within epineurium) and distinguish it from an extraneural cyst.

Closure. Failure to obtain hemostasis intraoperatively can lead to wound hematoma. Minimizing blood around the nerve can decrease the risks of extrinsic nerve compression or secondary fibrosis.

\section{Postoperative Regimen}

Adequate clinical and electrophysiological follow-up examinations and MR imaging are important to document complete treatment or to corroborate subclinical or clinical recurrences. ${ }^{2}$ Long-term follow-up care is necessary.

\section{Conclusions}

Diagnostic and surgical failures can be avoided if general principles are understood and recommended techniques are followed. In this two-part paper, we have detailed our current approach to patients with the most common form of intraneural ganglion, the peroneal nerve at the fibular neck originating from the superior tibiofibular joint. Understanding the principles that are the basis for the unified articular theory allows us to understand common modes of failure for peroneal intraneural ganglia and intraneural ganglia involving other nerves at other sites.

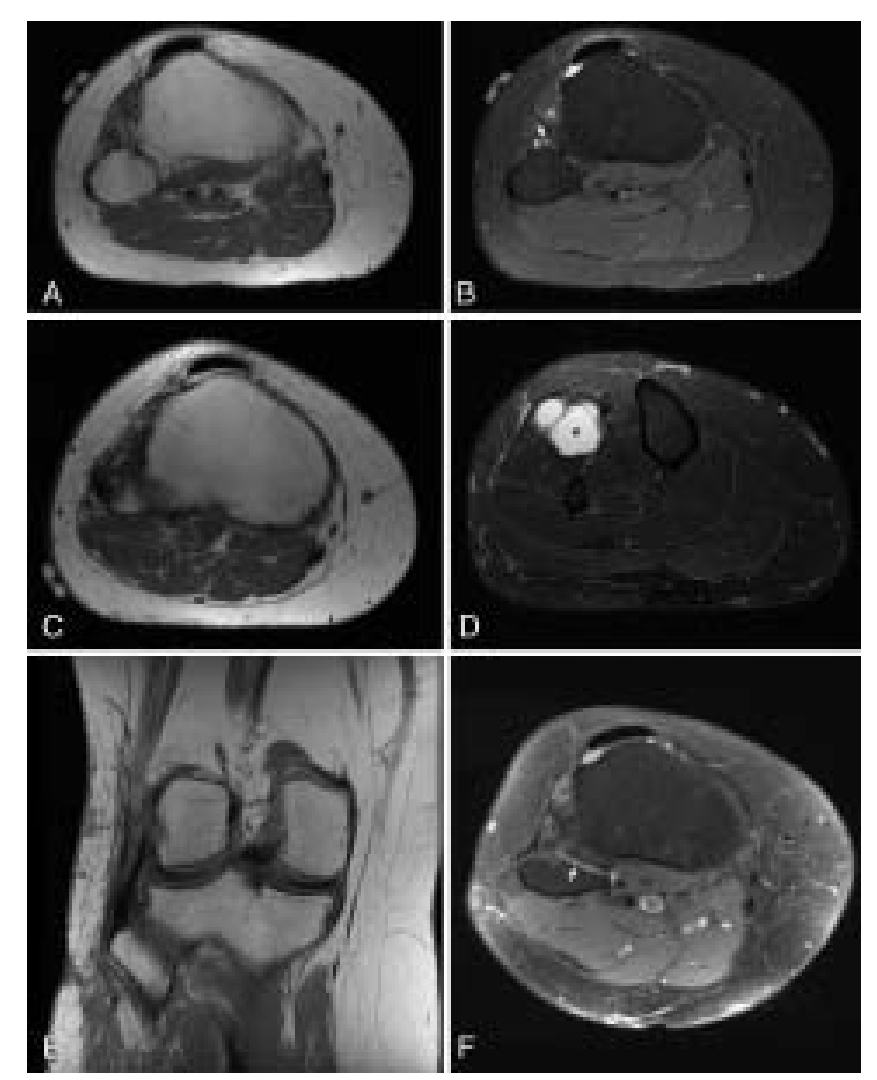

FIG. 7. Examples of extraneural recurrence after insufficient resection of the superior tibiofibular joint. The MR images highlight the superior tibiofibular joint. A and B: Preoperative imaging obtained in a patient with a combined peroneal intraneural and extraneural cyst. C and D: Initial postoperative imaging obtained in a patient after successful treatment of the peroneal intraneural cyst with decompression of the cyst and ligation and disconnection of the articular branch, but with extraneural recurrence due to incomplete resection of the superior tibiofibular joint. E and F: Postoperative imaging after successful extraneural cyst resection and resection of the superior tibiofibular joint. A: Axial T1-weighted MR image revealing the intact superior tibiofibular joint with mild degenerative changes. B: Axial T2-weighted FSE image with fat suppression revealing the origin of the intraneural ganglion cyst from the anterior portion of the superior tibiofibular joint prior to surgery (arrow). A large coexisting joint-related extraneural ganglion is not shown. C: Axial T1-weighted MR image demonstrating partial resection of the superior tibiofibular joint after the initial operation. D: Axial T2-weighted FSE image with fat suppression demonstrating a multiloculated cyst within the anterior compartment of the leg. This large recurrent extraneural cyst (asterisk) developed after initial surgery. The origin of the cyst from the anterior portion of the joint is visualized more proximally, but not on this image. E: Coronal T1-weighted MR image obtained after definitive surgery, demonstrating complete resection of the superior tibiofibular joint. F: Axial T2-weighted FSE image with fat suppression revealing more extensive postoperative changes with complete resection of the joint. There is a small amount of fluid present postoperatively, but no residual or recurrent cyst is seen (arrow).

\section{Acknowledgments}

We appreciate the opportunity to review a series of previously published MR images obtained in a patient treated by Dr. Kartik Krishnan (Dresden, Germany). We also appreciate the artistic excellence of David Factor (Rochester, Minnesota). 


\section{References}

1. Krishnan KG, Schackert G: Intraneural ganglion cysts: a case of sciatic nerve involvement. Br J Plast Surg 56:183-186, 2003

2. Spinner R: [In reference to the article: "Nerve compression caused by mucoid pseudocysts: arguments favoring an articular etiology (report of 23 cases)."] Rev Chir Orthop Reparatrice Appar Mot 91:492-494, 2005 (Fr)

3. Spinner RJ, Amrami KK: The Balloon Sign: Adn M, Hamlat A, Morandi X, Guegan Y (2006) Intraneural ganglion cyst of the tibial nerve. Acta Neurochir (Wien) 148:885-890. Acta Neurochir (Wien) 148:1224-1226, 2006 (Letter)

4. Spinner RJ, Amrami KK: Intraneural ganglion of the suprascapular nerve. Case report. J Hand Surg 31:1698-1699, 2006 (Letter)

5. Spinner RJ, Amrami KK, Kliot M, Johnston SP, Casañas J: Suprascapular intraneural ganglia and glenohumeral joint connections. J Neurosurg 104:551-557, 2006

6. Spinner RJ, Amrami KK, Wolanskyj AP, Desy NM, Wang H, Benarroch EE, et al: Dynamic phases of peroneal and tibial intraneural ganglia formation: a new dimension added to the unifying articular theory. J Neurosurg:in press, 2007

7. Spinner RJ, Atkinson JL, Scheithauer BW, Rock MG, Birch R, Kim TA, et al: Peroneal intraneural ganglia: the importance of the articular branch. Clinical series. J Neurosurg 99:319-329, 2003
8. Spinner RJ, Atkinson JL, Tiel RL: Peroneal intraneural ganglia: the importance of the articular branch. A unifying theory. $\mathbf{J}$ Neurosurg 99:330-343, 2003

9. Spinner RJ, Dellon AL, Rosson GD, Anderson SR, Amrami KK: Tibial intraneural Ganglia in the tarsal tunnel: is there a joint connection? J Foot Ankle Surg 46:27-31, 2007

10. Spinner RJ, Desy NM, Amrami KK: Cystic transverse limb of the articular branch: a pathognomonic sign for peroneal intraneural ganglia at the superior tibiofibular joint. Neurosurgery 59:157-166, 2006

11. Spinner RJ, Desy NM, Rock MG, Amrami KK: Peroneal intraneural ganglia. Part I. Techniques for successful diagnosis and treatment. Neurosurg Focus 22(6):E16, 2007

12. Spinner RJ, Mokhtarzadeh A, Schiefer TK, Krishnan KG, Kliot M, Amrami KK: The clinico-anatomic explanation for tibial intraneural ganglion cysts arising from the superior tibiofibular joint. Skeletal Radiol 36:281-292, 2007

Manuscript submitted March 30, 2007.

Accepted May 21, 2007.

Address reprint requests to: Robert J. Spinner, M.D., Mayo Clinic, Gonda 8S, Rochester, Minnesota 55905. email: spinner.robert@ mayo.edu. 